\title{
TAGUNG
}

\section{Die Fusionsthese in der Diskussion}

\author{
Andreas Hofmann*
}

Am 12. März wurde in Köln unter dem Titel „Europe Reloaded: Differentiation or $\mathrm{Fu}$ sion?" ein Workshop zu Fusions- und Differenzierungsprozessen in der Europäischen Union ausgerichtet und erste Ergebnisse eines Buchprojektes zur Würdigung der vom Kölner Politikwissenschaftler Wolfgang Wessels entwickelten „Fusionsthese“ präsentiert. Die Durchführung des Workshops erfolgte im Rahmen des vom 6. Rahmenforschungsprogramm der Europäischen Union geförderten Exzellenznetzwerks EU-CONSENT. ${ }^{1}$

Im Zentrum des Workshops wie des Buchprojektes ${ }^{2}$ steht eine kritische Diskussion der Fusionsthese als Erklärungsansatz zum Prozess der europäischen Integration. In der Einführung wurden Grundlagen dieses Ansatzes von Funda Tekin und Gaby Umbach referiert. Die Fusionsthese stellt den Integrationsprozess in den Kontext der Entwicklung der Staatlichkeit in Westeuropa seit der frühen Neuzeit. ${ }^{3}$ Sie erklärt die Integrationsbestrebungen der Mitgliedstaaten als Resultat des Versuches der gemeinschaftlichen Lösung von Problemen, die von den Mitgliedstaaten alleine nicht geleistet werden kann. In ihrem Streben nach der Beantwortung einer Reihe konstitutioneller Grundfragen suchen die Mitgliedstaaten nach einer adäquaten Problemlösungsebene

\section{Europe Reloaded: Differentiation or Fusion? EU-CONSENT Workshop}

Buchprojekt

Universität zu Köln in Kooperation mit dem Institut für Europäische Politik (IEP, Berlin)

Köln, 12. März 2008

\section{Welcome and Introduction}

Funda TEKIN, Gaby UMBACH, Universität zu Köln

The Evolution and Dynamics of the EU System: Theoretical, Political and Institutional Aspects

Chair: Anne FABER, Universität zu Köln

Fusion and Comitology

Thomas CHRISTIANSEN, European Institute of Public Administration

The big easy? Growth, differentiation and dynamics of EU enlargement policy 1973-2007

Barbara LIPPERT, Institut für Europäische Politik

Strengths of a fusing Europe

Lee MILES, Liverpool University

EMU, Economic Governance and Sustainable Development: Models and Trends

Chair: Gaby UMBACH, Universität zu Köln

Lisbon as economic governance: fusion or dif-fusion? Iain BEGG, London School of Economics

* Andreas Hofmann, M.A., Forschungsinstitut für Politische Wissenschaft und Europäische Fragen, Universität zu Köln.

1 Das Netzwerk behandelt Fragen zu den sich wechselseitig verstärkenden Effekten der Vertiefung und Erweiterung der Europäischen Union. Die Internetseite ist abrufbar unter: www.eu-consent.net (letzter Zugriff: 25.03.2008).

2 Die Publikation wird herausgegeben von Udo Diedrichs, Anne Faber, Funda Tekin und Gaby Umbach. Sie wird im Herbst 2008 in der Reihe Europäische Schriften des Instituts für Europäische Politik im Nomos Verlag, Baden-Baden, erscheinen.

3 Zum Einstieg in die Fusionsthese vgl. Wolfgang Wessels: Staat und (westeuropäische) Integration. Die Fusionsthese, in: Michael Kreile (Hrsg.): Die Integration Europas, PVS-Sonderheft 23, Opladen 1992, S. 36-61; Wolfgang Wessels: Die institutionelle Architektur des Verfassungsvertrags: Ein Meilenstein in der Integrationskonstruktion, in: Mathias Jopp/Saskia Matl (Hrsg.): Der Vertrag über eine Verfassung für Europa. Analysen zur Konstitutionalisierung der EU, Baden-Baden 2005, S. 45-85. 
und nach effizienten und demokratisch legitimierten Entscheidungsfindungsverfahren, ohne nationale Autonomie weitreichend abzugeben. Der Kölner Workshop stellte sich die Aufgabe, diesen Prozess in ausgewählten Politikbereichen der Europäischen Union zu betrachten, ihn mit Prozessen der Differenzierung $^{4} \mathrm{zu}$ kontrastieren und Erkenntnisse im Hinblick auf eine weiterführende Verfeinerung des Ansatzes zusammenzuführen.

\section{Das EU-System - Evolution und Dynamik}

Das Eröffnungspanel, geleitet von Anne $\mathrm{Fa}$ ber mit Beiträgen von Thomas Christiansen, Barbara Lippert und Lee Miles, fokussierte auf eine Gesamtsicht der Evolution des EUSystems im Lichte des Fusionsansatzes. Hier wurde festgehalten, dass die Fusionsthese in erster Linie eine Erklärung für die Stabilität der Evolution des EU-Systems bietet. Als Voraussetzung für diese Stabilität identifizierten die Referenten eine ,Sub-Optimalität“ des Systems, die verhindere, es genauer zu klassifizieren. Anstatt einer eindeutigen Entwicklung in Richtung einer bestimmbaren Finalität seien vielmehr gerade die Verschränkungen zwischen supranationalen und intergouvernementalen Elementen des Systems wesensbestimmend. Die Verschränkung fördere die Stabilität eines ,dritten Wegs ‘ der Systementwicklung zwischen der Nachfrage nach supranationalen Lösungen auf der einen und der Skepsis gegenüber einer föderalen finalité auf der anderen Seite. Dieses Resultat wird als Ausdruck der Leistungsorientierung der verantwortlichen Politiker gewertet, die die Handlungsfähigkeit des Staates durch übernationale Integration $\mathrm{zu}$ wahren suchen, ohne gleichzeitig eigene Einflussmöglichkeiten zu verlieren. Zugleich sei das resultierende Gefüge von einer Undeutlichkeit der Kompetenzabgrenzung zwischen den involvierten Ebenen gekennzeichnet.
European Monetary Union and enhanced cooperation

Jean Victor LOUIS, Prof. em. Université Libre de Bruxelles

The potential for integration of , sustainable development ${ }^{\circ}$

Hartmut MARHOLD, Centre International de Formation Européenne

The External Dimension of the „new“ EU: Fused or Differentiated?

Chair: Udo DIEDRICHS, Universität zu Köln

EU's projection of security: peace missions as a tool both for fusion and differentiation

Gianni BONVICINI, Istituto Affari Internazionali

European Neighbourhood Policy and internal and external security

Gunilla HEROLF, Stockholm International Peace Research Institute

Common Foreign and Security Policy and European Security and Defence Policy - Fusion trends and future perspectives

Elfriede REGELSBERGER, Mathias JOPP, Institut für Europäische Politik

Europe Reloaded: Differentiation or Fusion? Revisiting Workshop Results

Chair: Wolfgang WESSELS, Universität zu Köln

Introduction: Anne FABER, Udo DIEDRICHS, Universität zu Köln

Panel Participants: Gunilla HEROLF, Stockholm International Peace Research Institute; Hartmut MARHOLD, Centre International de Formation Européenne; Lee MILES, Liverpool University

\section{Conclusions}

Anne FABER, Udo DIEDRICHS, Universität zu Köln

Aus den ersten Panelbeiträgen ergab sich folgendes Bild: Zentrale Aussagen der Fusionsthese in Bezug auf institutionelle Prozesse, wie sie insbesondere Wessels in einer Reihe von Artikeln seit der Mitte der Neunzigerjahre formulierte, seien durch Entwicklungen des letzten Jahrzehnts bestätigt worden. So sei

4 Differenzierung ist in diesem Zusammenhang zu verstehen als Ungleichzeitigkeit von Integrationsschritten zwischen verschiedenen Mitgliedstaaten und die daraus resultierende Uneinheitlichkeit der Integrationstiefe innerhalb des EU-Systems. 
eine zunehmende Verschmelzung von legislativen und administrativen Prozessen festzustellen, die eine wachsende Politisierung administrativer Vorgänge zur Folge habe. Bestätigt wurde auch die Erwartung einer zunehmenden Nachfrage nach Beteiligung an Politikgestaltungsprozessen auf der europäischen Ebene von Seiten regionaler, nationaler und europäischer Akteure. Gleichzeitig sei eine wachsende Kluft zu beobachten zwischen europäisch integrierten politischen Akteuren und solchen, die keine europäische Anbindung vorweisen können.

Hingegen sei die beobachtete Differenzierung innerhalb des Systems der divergierenden Leistungsfähigkeit der Mitgliedstaaten zuzuschreiben. Dabei seien bestimmte, von Miles als ,Fusions-Cluster' bezeichnete Gruppen zu identifizieren, innerhalb derer sich unterschiedlich integrierte Mitgliedstaaten weiterhin institutionell verschmelzen. Diese enge Bindung zwischen Mitgliedstaaten in unterschiedlichen Integrationsstadien, so wie sie etwa in Mechanismen der Beteiligung des Vereinigten Königreichs und Dänemarks an der Währungspolitik der Euro-Zone und des Nicht-EUMitglieds Norwegen am Schengenraum zu beobachten sei, trage maßgeblich zur Stabilität des Systems bei. Die zu konstatierende Divergenz werde durch einzelne Erweiterungsrunden verstärkt. Kritisch sei in diesem Zusammenhang jedoch festzuhalten, dass das theoretische Erklärungsangebot hinsichtlich der Erweiterungen auch im Fall der Fusionsthese relativ schwach sei. Im Bezug auf die Nachbarländer der Union stellte das Instrument der Erweiterung über lange Zeit das einzige Instrument der Strukturierung der Beziehungen dar. Diese ,Erweiterungsdoktrin “ sei nach den letzten Erweiterungsrunden zunehmend unter Druck geraten; alternative Instrumente gewännen an Bedeutung. Die Europäische Union übernehme im Zuge ihrer Erweiterungspolitik eine neuartige Rolle als Garant der Stabilität in den Kandidatenländern.
Wirtschafts- und Sozialpolitik als Fusionsprozess

Das nachfolgende, von Gaby Umbach geleitete Panel, mit Beiträgen von Jean Victor Louis, Iain Begg und Hartmut Marhold, behandelte Prozesse der Fusion und Differenzierung in den Bereichen der Wirtschafts- und Sozialpolitik. Die Referenten identifizierten die Währungspolitik als gutes Beispiel für einen Fusionsprozess, in dem die Gleichzeitigkeit von supranationalen (Wirtschafts- und Währungsunion) und intergouvernementalen (Stabilitäts- und Wachstumspakt) Elementen innerhalb einzelner Politikbereiche zum Ausdruck kommt. Darüber hinaus sei im Bereich der Währungspolitik eine ausgeprägte Tendenz zur Differenzierung zu erkennen, wobei jedoch Brücken zwischen den ,ins' und den ,outs' existierten. Verwandte Politikbereiche, wie Steuerpolitik und Beschäftigung, könnten möglicherweise das Potenzial weiterreichender differenzierter Kooperation bergen.

Auch die Offene Methode der Koordinierung (OMK) als Instrument der Zusammenarbeit in neuen Politikbereichen im Rahmen der Lissabon-Strategie könne als Kompromiss zwischen supranationalen und intergouvernementalen Modi der Politikgestaltung gesehen werden. In diesem Zusammenhang sei ein ,Mega-Prozess' zu identifizieren, der eine Annäherung nationaler Zielsetzungen in den behandelten wirtschafts- und sozialpolitischen Bereichen - als Antwort auf externe Prozesse der Globalisierung - zur Folge habe. Von besonderer Bedeutung seien diesbezüglich Lernund Sozialisationsprozesse, die aus dem Koordinationsprozess in nationale Projekte, diffundierten'. Jedoch blieben die Verbindungen zu makropolitischen Steuerungsinstrumenten unklar. Betont wurde hier die Notwendigkeit eines umfassenderen ,Policy-Mix ‘ zur Beantwortung der globalen Herausforderungen, aber auch die Gefahr des Überschwappens der weniger verbindlichen OMK in bereits vergemeinschaftete Bereiche (so beispielsweise in der Umweltpolitik) und somit die Risiken eines Rückbaus von integrierten Politiken. 
In seinem Beitrag vertrat Marhold die Einschätzung, dass das mit der Unterzeichnung des Vertrags von Lissabon - zumindest rhetorisch - eingeleitete Ende des institutionellen Reformprozesses gleichzeitig den Raum für neue inhaltliche Projekte eröffne. Allerdings sei die Vorstellung eines ,Europe des projets - im Sinne vielfältiger Projekte - nicht realistisch. Vielmehr müsse ein zentrales Projekt gefunden werden, das einzigartig und identifizierbar den Integrationsprozess über einzelne Politikbereiche hinaus tragen kann. Als besonders erfolgversprechend schlug Marhold das Projekt der ,nachhaltigen Entwicklung beziehungsweise der , neuen Nachhaltigkeitsstrategie ' vor. Mit konkreter Substanz gefüllt verspräche es durch die Einbeziehung verschiedener Politikbereiche und Akteure ein besonderes Integrationspotenzial. In diesem Zusammenhang gewinne zudem die Frage politischer Führung an Relevanz, da gerade die Implementierung neuer Politikbereiche einer solchen bedarf. Als Vorteil des Fusionsansatzes zur Erklärung solcher Prozesse wurde sein Fokus auf Akteure verschiedener Ebenen, regional wie national und übernational betont.

\section{Fusionsthese und auswärtiges Handeln}

Das dritte Panel, geleitet von Udo Diedrichs mit Beiträgen von Elfriede Regelsberger, Mathias Jopp, Gianni Bonvicini und Gunilla Herolf, widmete sich dem auswärtigen Handeln der Union. Auch in diesem Bereich, so betonten die Referenten, sei eine dem Fusionsprozess entsprechende zunehmende Verschmelzung von Politikgestaltung auf der nationalen und europäischen Ebene festzustellen, obwohl in der Gemeinsamen Außen- und Sicherheitspolitik und der Europäischen Sicherheitsund Verteidigungspolitik weiterhin ein intergouvernementaler Modus vorherrsche. $\mathrm{Zu}$ beobachten sei neben einer Zunahme außenpolitischer Instrumente insbesondere eine $\mathrm{Zu}$ nahme an Akteuren: Eine neue Kategorie ,fusionierter' Akteure steche hervor, die sich durch eine unklare Abgrenzung von Kompetenzen auszeichnen - so etwa der neu konzipierte Hohe Vertreter der Union für Außen- und Sicherheitspolitik oder der Europäische Auswärtige Dienst. Europäische Institutionen tragen zu einer Verschmelzung verschiedener nationaler Wahrnehmungen bei, die sich in einem ,Fusionskreislauf“ wiederum auf die Ausformung dieser Institutionen auf der europäischen Ebene auswirken. Auch in diesem Prozess lassen sich Differenzierungstendenzen identifizieren. Diese verlaufen vornehmlich entlang der zivil-militärischen Kapazitäten des Krisenmanagements der Mitgliedstaaten, so etwa in der mit dem Lissabonner Vertrag ermöglichten „ständigen strukturierten Zusammenarbeit“. Zentral werde in diesem Bereich, so die Referenten, die zukünftige Suche nach interner und externer Kohärenz des auswärtigen Handelns der Union.

\section{Resümee und offene Fragen}

Das abschließende Panel, geleitet von Wolfgang Wessels mit Diskussionsbeiträgen von Gunilla Herolf, Hartmut Marhold und Lee Miles, resümierte die Ergebnisse des Workshops und stellte die zukünftigen Möglichkeiten und Herausforderungen des Fusionsansatzes zur Diskussion. Als grundlegende Frage des Forschungsansatzes formulierten die Beteiligten: Wie kann der Entwicklungsprozess der Union von einer Gemeinschaft von sechs Mitgliedstaaten mit einer engen Ausrichtung auf den Montanbereich hin $\mathrm{zu}$ einer gesamteuropäischen Union der 27 mit einer staatsähnlichen Agenda analysiert, erklärt und bewertet werden? Der Fokus des Fusionsansatzes, so betonten die Referenten, liege mit dieser Fragestellung auf einer langfristigen Perspektive der Systementwicklung. Ein solcher Makro-Ansatz erscheine den involvierten komplexen Zusammenhängen angemessen. Die entsprechenden Untersuchungen müssen sich daher auf mehreren Ebenen vollziehen. Die Analyse des Einigungsprozesses werde so eingebettet in die Entwicklung der Staatlichkeit über einen weit über die Pariser Verträge zurückreichenden Zeitraum. Im Zuge dieses Prozesses komme es zu einer Transformation nicht nur des politischen Systems der Union, sondern auch - und gerade - zu einem Wandel der 
Staatlichkeit auf der nationalen Ebene. Der Europäische Rat als ,konstitutioneller Architekt ${ }^{\circ}$ der Union stelle, so betonte Wessels in seinem Beitrag, in dem geschilderten Prozess das zentrale Bindeglied zwischen den interdependenten Entwicklungen auf nationaler und europäischer Ebene dar. Er bestimme als ,Institution der Mitgliedstaaten " diese Entwicklungen weitgehend und verdiene daher die besondere Aufmerksamkeit des Fusionsansatzes. Die geschilderte Situation sei gekennzeichnet von einer Reihe konstitutioneller Dilemmata, deren Beantwortung durch die Mitgliedstaaten ein bestimmtes Muster der Integrationsentwicklung erkennen ließe - einen Prozess von der Koordinierung einzelner Politikbereiche zu Kooperation zwischen den Mitgliedstaaten und schließlich der Vergemeinschaftung dieser Bereiche. Diese Annahme werfe jedoch die Frage auf, warum manche Politikbereiche vollständig diesem Muster folgen, andere jedoch nur begrenzt.

Im Laufe der Diskussion wurden das besondere Potenzial der Fusionsthese, aber auch ihre konzeptionellen Schwächen deutlicher. Notwendig sei, so das Resümee, eine Präzisierung der potenziellen Umstände, die die Erwartungen der Fusionsthese falsifizieren könnten. Klärungsbedürftig sei ferner, welche Prozesse als ,Fusion * $\mathrm{zu}$ bezeichnen seien und welche nicht. Eine Feinabstimmung des Konzepts werde die Debatte bereichern und die Güte der Erkenntnisse erhöhen. In diesem Zusammenhang formulierten Anne Faber und Udo Diedrichs in ihren Schlussbemerkungen vier weiterführende Forschungsfragen, die die Diskussionen des Tages aufgeworfen hatten: An welchem Punkt verlässt der Prozess der Differenzierung die Annahmen des Fusionsansatzes? Wie können externe Akteure und externe ,Schocks' in den Ansatz eingebunden werden? Inwiefern beeinflussen Erweiterungsrunden den Fusionsprozess? Wie ist die Notwendigkeit politischer Führung mit dem Fusionsansatz zu vereinbaren?

Viele Beiträge aus dem Kreise der WorkshopTeilnehmer lassen auf eine Beantwortung dieser zentralen Fragen und eine fruchtbare Weiterentwicklung des Fusionsansatzes hoffen.

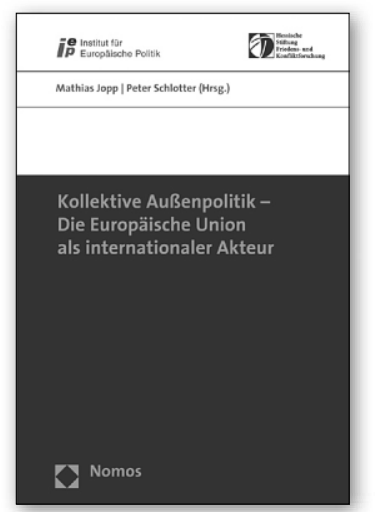

\section{Kollektive Außenpolitik - Die Europäische Union als internationaler Akteur}

Herausgegeben von Mathias Jopp und

Peter Schlotter

2. unveränderte Auflage 2008, 397 S., brosch., 39,- $€$, ISBN 978-3-8329-3360-9

(Europäische Schriften, Bd. 86)

"Insgesamt ist ein für Lehrende und

Studierende zu empfehlender Sammelband

entstanden." Siegfried Schwarz, Berlin, Welt Trends 58/08

Bitte bestellen Sie bei Ihrer Buchhandlung

oder bei Nomos | Telefon 07221/2104-37 | Fax -43

www.nomos.de | sabine.horn@nomos.de

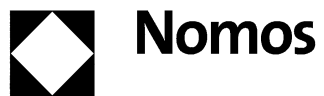

